\title{
Testing of Dynamic Models for Stability Studies
}

\author{
Karel Máslo ${ }^{1)}$ and Tomáš Hába ${ }^{2)}$ \\ 1) 2) Transmission System Analysis Department, ČEPS, a.s., Prague, Czech Republic \\ e-mail: ${ }^{1)}$ maslo@ceps.cz, ${ }^{2)}$ haba@ceps.cz
}

\begin{abstract}
This paper deals with turbine-governor and excitation system dynamic models which are suitable for dynamic stability assessment of a transmission system. ENTSO-E uses the Common Grid Model Exchange Specification (CGMES) to facilitate the exchange of operational and grid planning data among transmission system operators. CGMES also includes a detailed description of the models needed to perform dynamic stability studies. These models are compared with the models implemented in the network simulator MODES.
\end{abstract}

Keywords - dynamic stability, excitation and turbinegovernor models, power system stabilizer.

\section{INTRODUCTION}

The dynamic stability (rotor angle, frequency and voltage) is an important part of the power system secure operation. A time domain simulation on a detailed dynamic power system model is traditionally used for the dynamic stability assessment.

The power system dynamic is investigated as a response to different network disturbances like shortcircuits and island operation. The basic requirement for such investigations is the use verified and credible dynamic models of power system equipment especially excitation and turbine-governor models. Before using dynamic such models for simulation calculations on a real system, it is appropriate to test dynamic behavior of the models in a simple network to get response to specific disturbances. For this purpose, ENTSO-E working group System Protection and Dynamics (SPD) defined the testing methodology [1]. Three simple tests are presented in the report [1]. Two tests are used in this paper to present dynamic response of several generic dynamic models. These models are selected from the Common Grid Model Exchange Specification (CGMES) - it is a standard for the exchange of operational and grid planning data among ENTSO-E transmission system operators (TSO).

The paper is structured as follows. The test methodology is described in section II. The turbinegovernor and excitation systems dynamic models are introduced in the sections III and V. Simulation results are presented in the sections IV and VI. Finally, the conclusion is drawn in section VII.

\section{Test CAse Methodology}

ENTSO-E SPD defined elementary test cases to compare results obtained from different simulation tools. Fig. 1 gives the single line diagram used as the primary test configuration. Tested synchronous unit with a generic name GEN is connected through unit transformer T-GEN to an infinite source GRID.

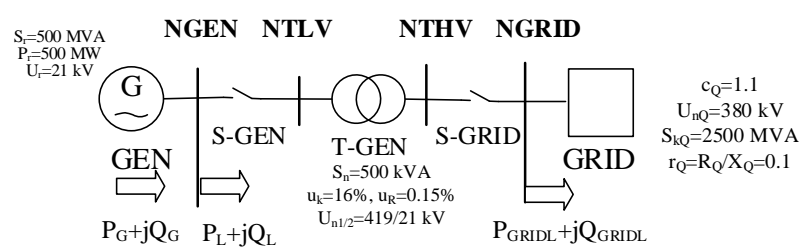

Fig. 1. Single line diagram for the test configuration (according to [1]).

Report [1] defines three test cases for time domain simulation to get representative results of the dynamic behavior of the synchronous unit and its control systems. The case 1 tests the dynamic performance of the automatic voltage control system. The test case 2 provides the turbine- governor response to the load change in an island operation. The test 3 reveals unit dynamic behavior during and after three-phase short-circuit that occurred at the high voltage side of the transformer T-GEN. We use only case 2 and 3 for the purposes of this paper.

The switch S-GEN is opened during the test case 2 according to Fig. 1, which simulates the island operation. Local active load $P_{\mathrm{L}}$ reaches $80 \%$ from nominal generator active power rating. Reactive load is $Q_{\mathrm{L}}=0$ and the terminal voltage of the generator is nominal ( 1 per unit). $P_{\mathrm{L}}$ is increased by $6.25 \%$ from the initial value at time $t=1 \mathrm{~s}$. It corresponds to $0.05 \mathrm{pu}$ related to the generator active power rating.

The test case 3 reveals the dynamic behavior of the synchronous machine model with its whole control system in operation during and after a three-phase short-circuit that occurred at the high voltage side of the transformer depicted in Fig. 1. Both S-GEN switch and S-GRID switch are closed. The generator is loaded with its nominal active power $P_{\mathrm{G}}$ and the node voltage of NGRID is being kept at the value of $399 \mathrm{kV}$. Furthermore, the constant impedance load GRIDL is modeled as active and reactive power consumption with the value of the load flow $475+$ i76 MVA. The three-phase short-circuit occurs at the event time $t=100 \mathrm{~ms}$. After the fault duration of $100 \mathrm{~ms}$ the initial system conditions are restored.

\section{TURBINE-GOVERNOR MODELS}

The standard CGMES includes a wide range of turbinegovernor and excitation system models used by various computational programs. Reference [2] has analyzed, sorted and compared these models with the models used in the network simulator MODES.

The following figures compare selected models from the CGMES standard with the turbine-governor models implemented in the library of MODES (see [3] for more information). Examples of steam, hydro and gas turbines were chosen for demonstration.

Detailed description of CGMES models (including typical parameters) is available in [4]. 


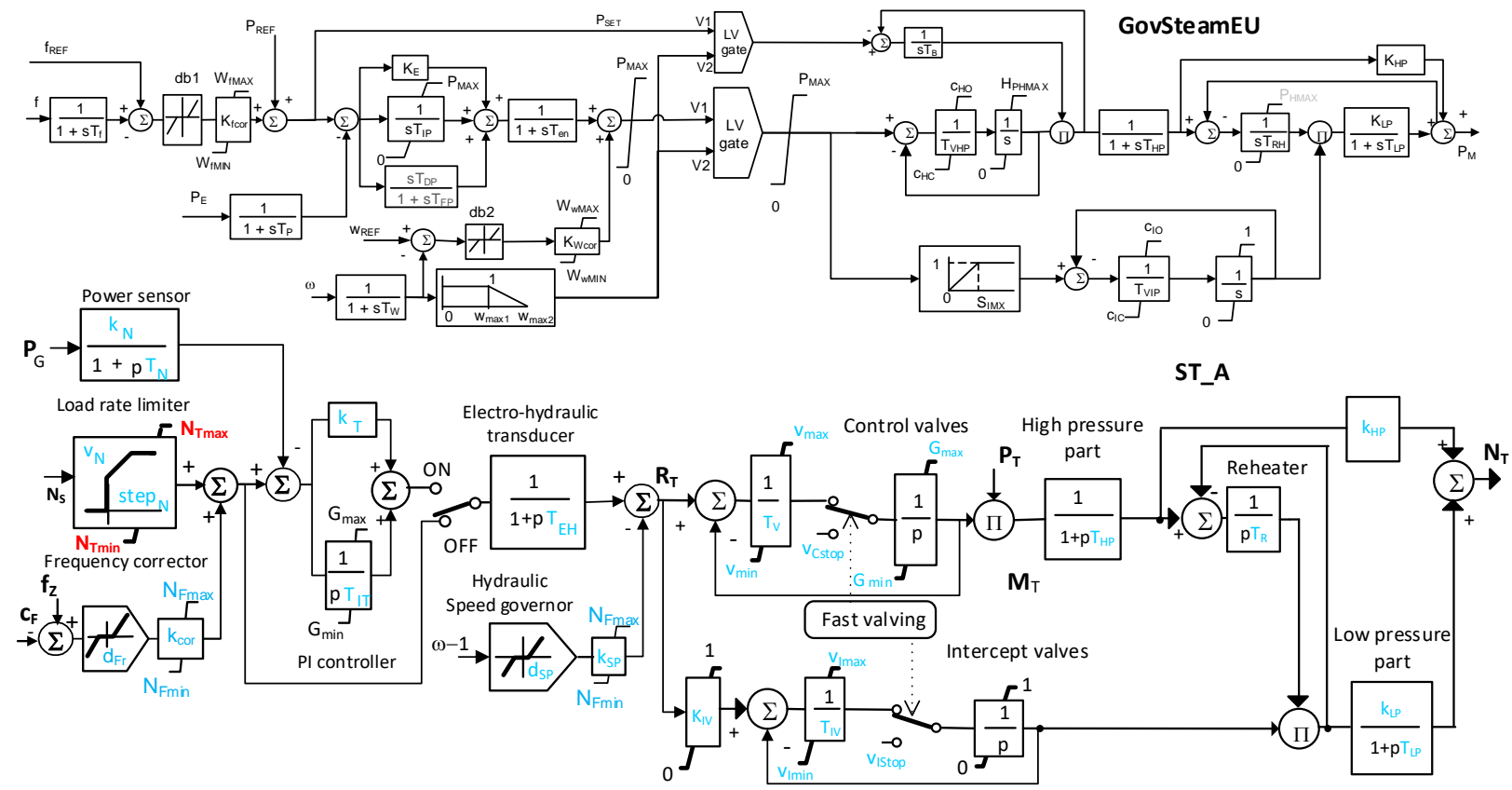

Fig. 2. Block diagram of GovSteamEU model (upper part according to [4]) and the corresponding model ST_A from the MODES library.
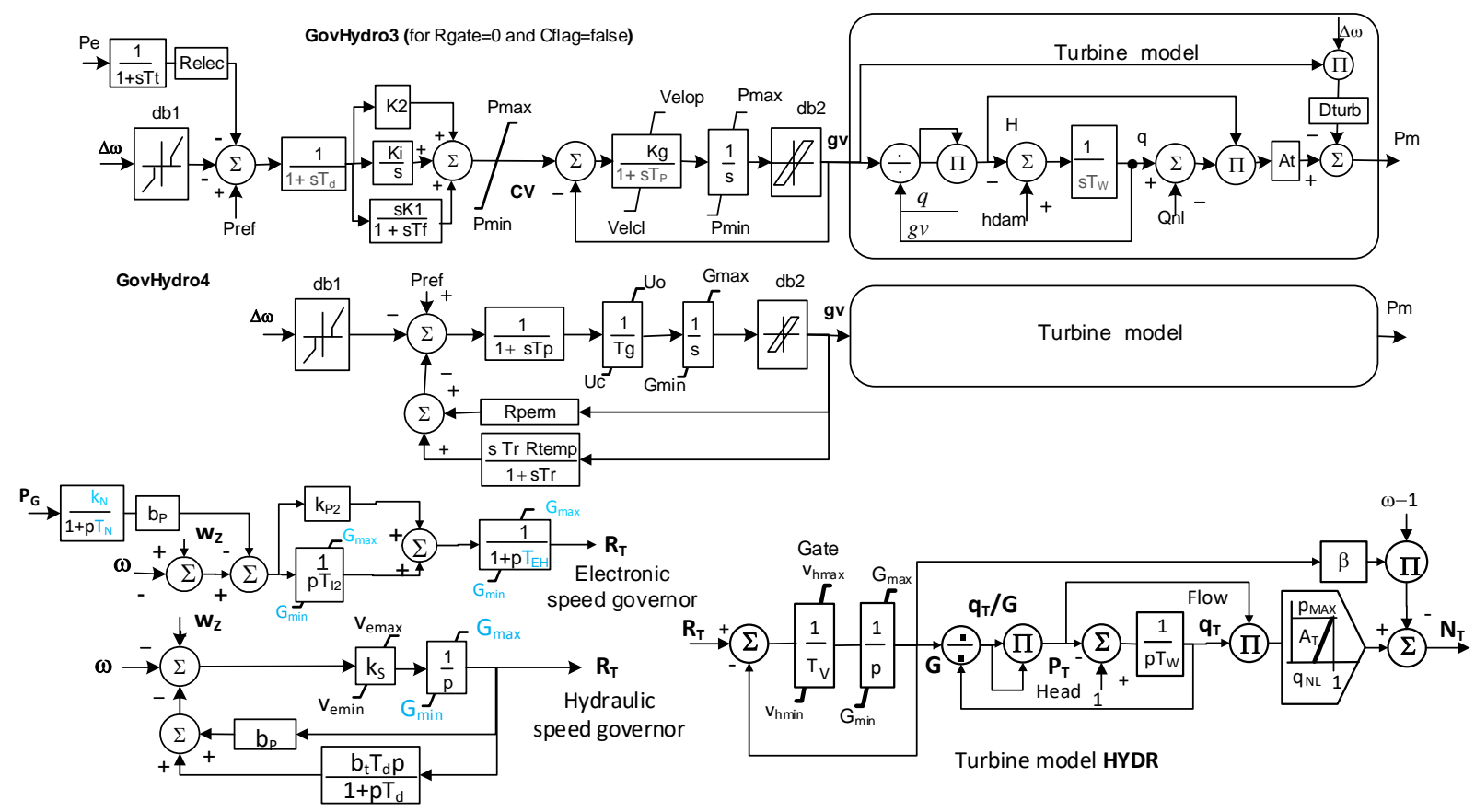

Fig. 3. Block diagram of HovHydro3 and GovHydro4 models (upper part according to [4]) and the corresponding model HYDR from MODES. 


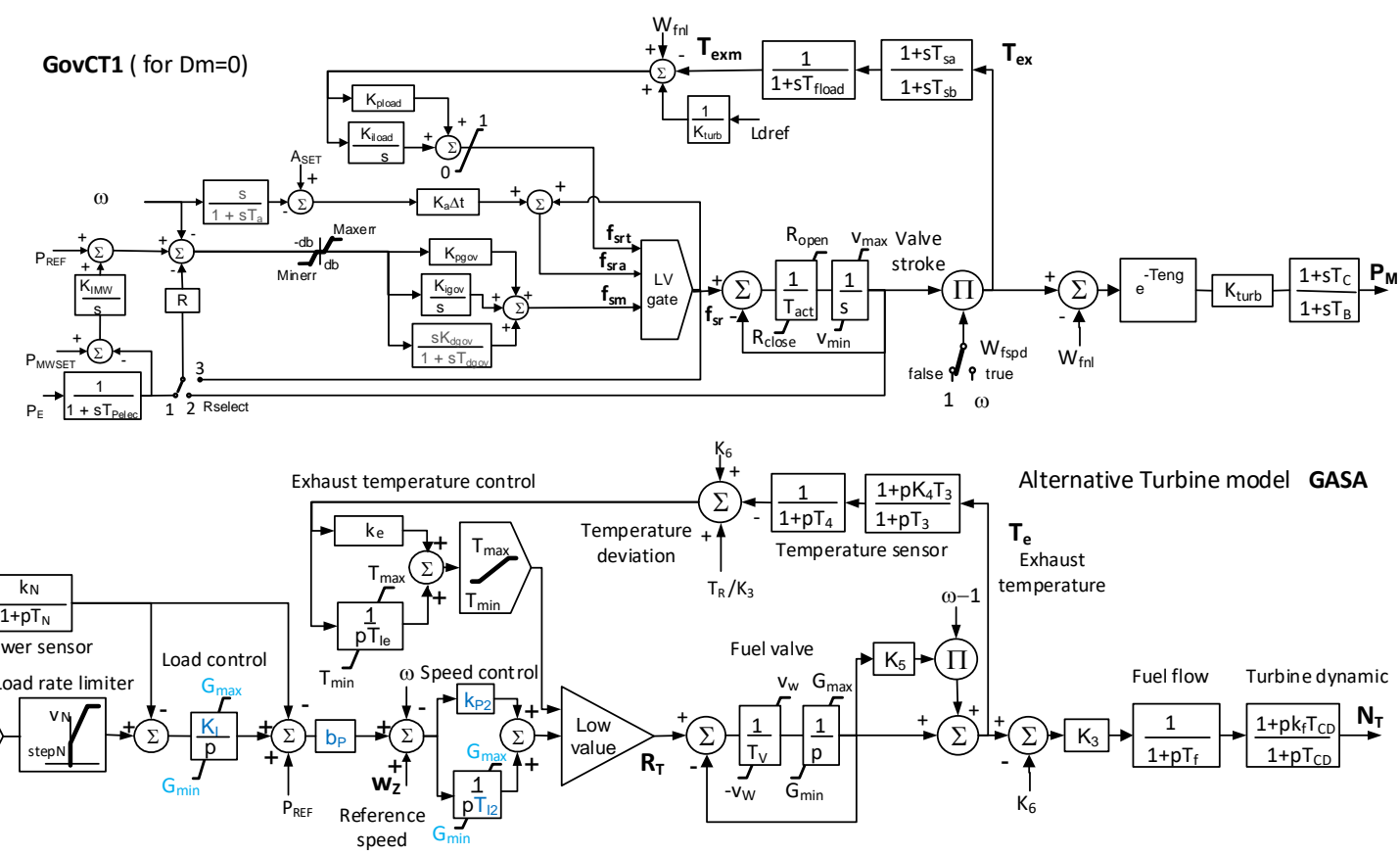

Fig. 4. Block diagram of GovCT1 model (upper part - simplified and modified according to [4]) and the corresponding model GASA from MODES.

\section{A. Steam Turbine Model}

The model GovSteamEU, depicted in the upper part of Fig. 2. allows to simulate the power control mode, which is the most common control mode for synchronous interconnection and provides some ancillary services (primary and load frequency controls). This model also considers changes in admission pressure (using one time constant $T_{\mathrm{B}}$ ) in a simplified way.

The detailed model of the steam turbine ST_A (implemented in MODES) along with the universal controller model is shown in the lower part of Fig. 2. Parameter $N_{\mathrm{S}}$ enables to connect the unit to the remote load frequency control. The frequency correction enables to provide the primary frequency control.

\section{B. Hydro Turbine Model}

The GovHydro4, depicted in the upper part of Fig. 3, represents a hydro turbine model with a traditional mechanical-hydraulic governor, consisting of a hydraulic pilot valve, main servo, and dashpot temporary droop.

The block diagram GovHydro3 represents a simplification of the original diagram with the consideration that the value of the parameter $R_{\text {gate }}$ is 0 and $C_{\text {flag }}$ is true, which indicates that the PID control is active.

The non-linear dependence between the gate position $G_{\mathrm{v}}$ and turbine power $P_{\mathrm{gv}}$ can be neglected for the angle and frequency stability calculations since this nonlinearity was not considered in both block diagrams GovHydro3 and GovHydro4.

A compatible model HYDR is shown in the lower part of Fig. 3. The hydraulic head is considered to be nominal $\left(H_{\mathrm{dam}}=1\right)$. Two types of speed governors are available in the MODES. The first is an electronic speed governor with a structure corresponding to the GovHydro3 model. The second is a hydraulic speed governor with a structure corresponding to the GovHydro4 model. Both are shown in Fig. 3.

\section{Gas Turbine Model}

The block diagram GovCT1, which is depicted in the upper part of Fig. 4, represents a simplification of the original diagram with the consideration that the value of parameter $D_{\mathrm{m}}$ is 0 . The model GovCT1 has three control loops for:

1. main governor,

2. exhaust temperature control,

3. acceleration control.

The turbine is controlled by the loop with the lowest value at the output. Smooth transition between power control and exhaust temperature control ensures integration elements ( $K_{\text {iload }}$ and $K_{\text {igov }}$ ). The main governor can work in several modes, depending on the $R_{\text {select }}$ Switch. For values 2 and 3 it has a similar character to the speed governor of the hydro turbine. The proportional and isochronous speed controls are available for values 1 and 0 . Additionally, the controller allows remote control of power and changes the base opening $P_{\text {ref }}$ to adjust the power output to $P_{\text {MWset. This function was blocked }}$ $\left(K_{\mathrm{imw}}=0\right)$ in the step test. The turbine output may depend on the speed through the fuel supply (depending on the $W_{\text {fspd }}$ switch).

A compatible model GASA is depicted in the lower part of Fig. 4. It corresponds to the GovCT1 model with neglecting of the transport delay $T_{\text {eng }}$ (which does not occur in the operation of gas turbines) and the acceleration control loop. An alternate speed controller allows both proportional and isochronous speed control (corresponding to the options $R_{\text {select }} 1$ and 0 ) as well as a change in the basic opening by the remote control. 


\section{TEST CASE 2 - SimUlation Results}

This part summarizes the results of the dynamic simulations according to the test case 2 . The simulations were performed in the network simulator MODES.

Following charts show the turbine power $N_{\mathrm{T}}$ response to step change of generator power $P_{\mathrm{G}}$ and the waveform of speed $n$.

\section{A. Steam Turbine}

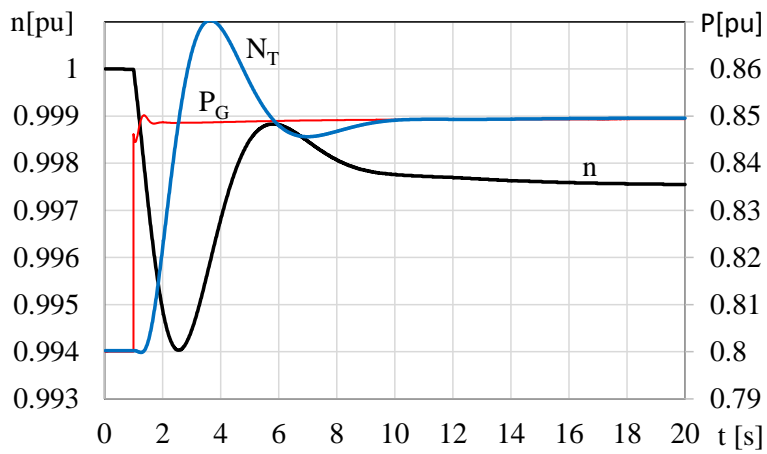

Fig. 5. Steam turbine response to the load step change.

It is seen almost step change in generator active power $P_{\mathrm{G}}$, which causes a decrease in speed $n$. The energy is taken from inertias during this period. Then the turbine governor reacts and increases required turbine power $N_{\mathrm{T}}$. The steam turbine provides the fastest response, even though the power slightly drops from the start due to the effect of the power controller. However, then the turbine increases the power quickly because it acts both as a speed governor and frequency corrector in the power controller. The change of speed $n$ depends on the frequency correction gain $K_{\mathrm{COR}}$.

\section{B. Hydro Turbine}

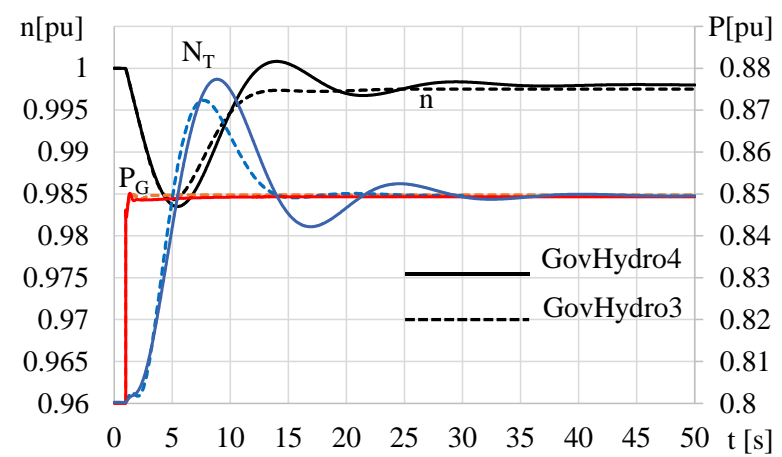

Fig. 6. Hydro turbine response to the load step change.

The hydro turbine gives the slowest response (due to preventing a water hammer effect) and hence the most significant speed deviation. The speed deviation depends on the permanent speed droop $b_{\mathrm{P}}$.

\section{Gas Turbine}

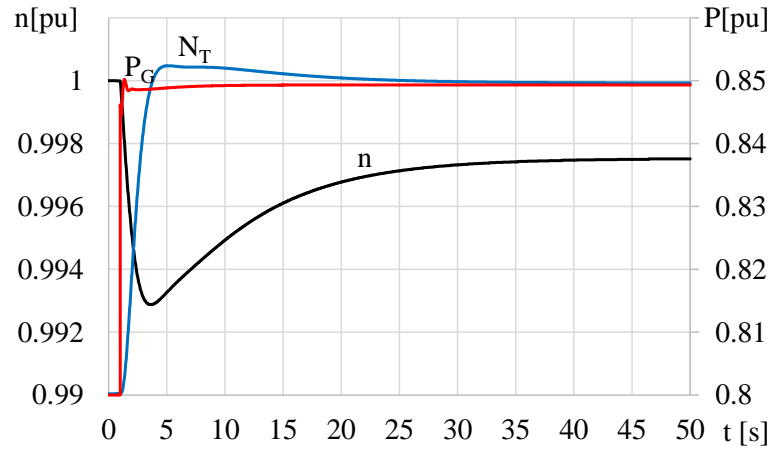

Fig. 7. Gas turbine response to the load step change.

The gas turbine provides a fast response, but the control process lasts longer than for the steam turbine. As already mentioned, the parameter $K_{\mathrm{iMw}}$ was set to zero. Otherwise, the load control would restore the initial turbine output and the frequency in the island would steadily decrease. The change of speed is also dependent on the permanent speed droop as in the hydro turbine case.

The following figure compares speed waveforms for all tested turbine-governor models.

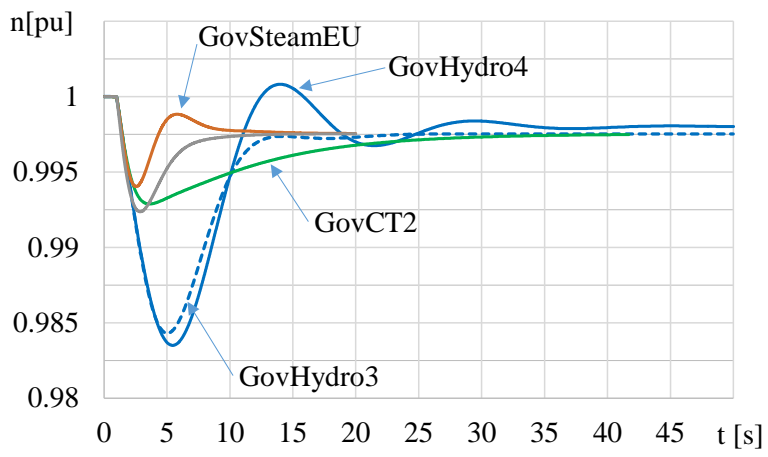

Fig. 8. Comparison of the speed waveforms for generic turbine models.

The best course is achieved by the steam turbine model GovSteamEU, followed by the gas turbine model GovCT2 and finally the hydro turbine GovHydro3. These models have steady state speed deviation $\Delta n=0.0025 \mathrm{pu}$. The model GovHydro4 has a slightly smaller deviation due to the turbine gain $A_{\mathrm{t}}$ and the lack of feedback from the electric power $P_{\mathrm{G}}$ in the governor. The grey waveform belongs to the turbine model TGOV1 from the reference [1].

\section{EXCITATION SYSTEM MODELS}

Three generic models of excitation systems were selected to simulate the dynamic response of the synchronous unit to the three-phase short-circuit.

The following figures show block diagrams of these models according to the CGMES and corresponding models implemented in the MODES. 

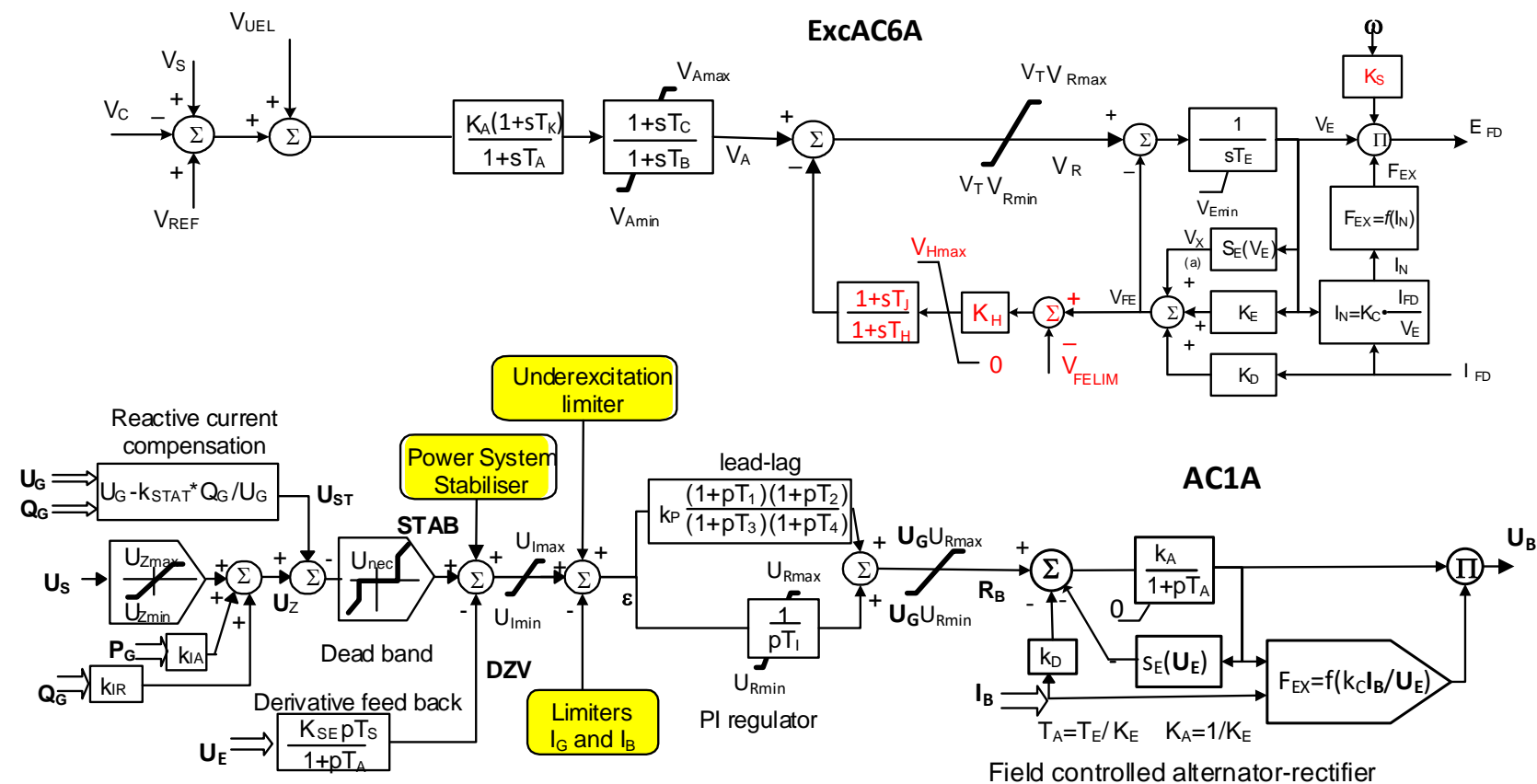

Fig. 9. Block diagram of ExcAC6 model (upper part according to [4]) and the corresponding model AC1A from MODES.

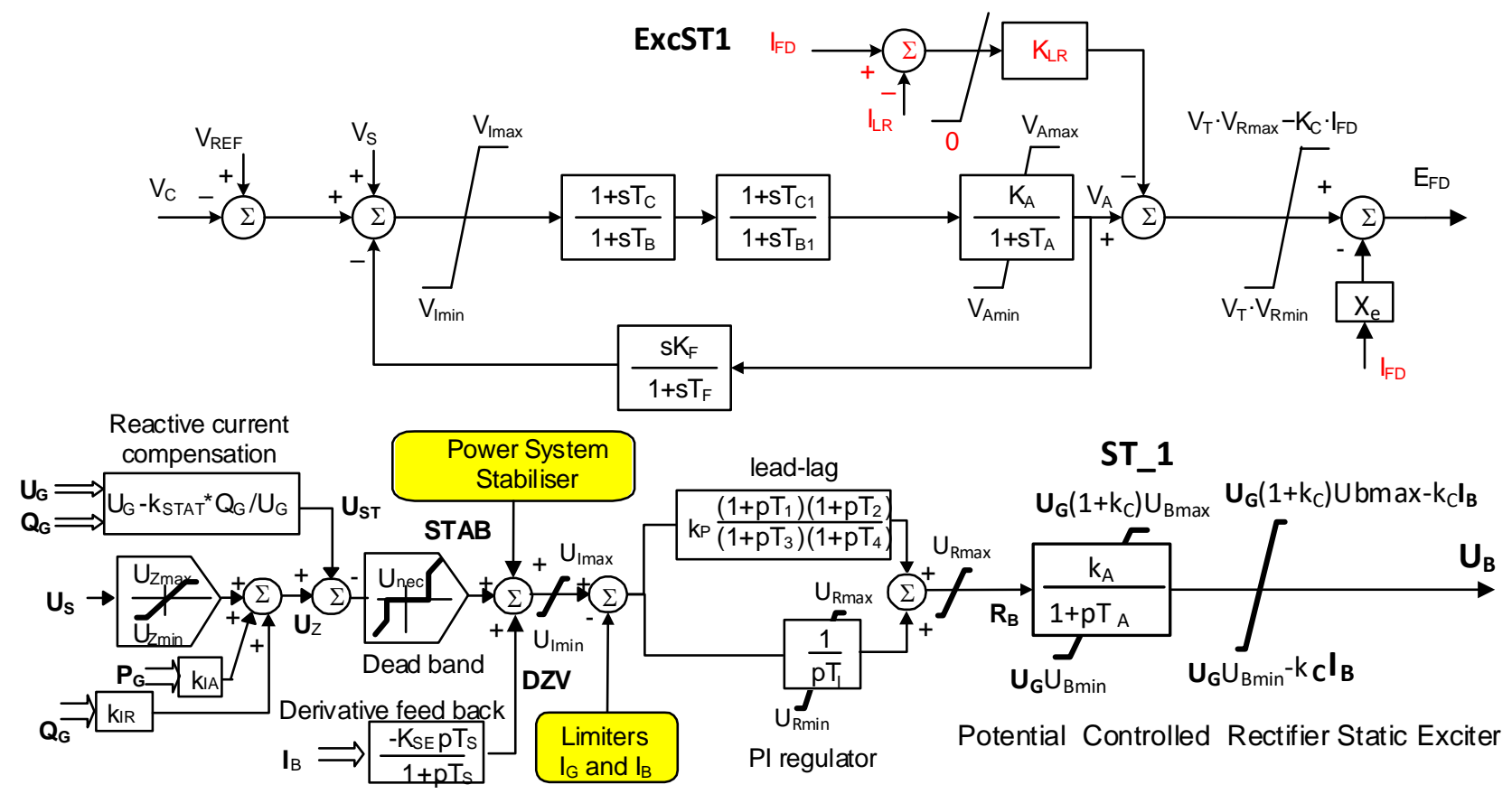

Fig. 10. Block diagram of ExcST1A (upper part according to [4]) and the corresponding model ST_1 from MODES. 


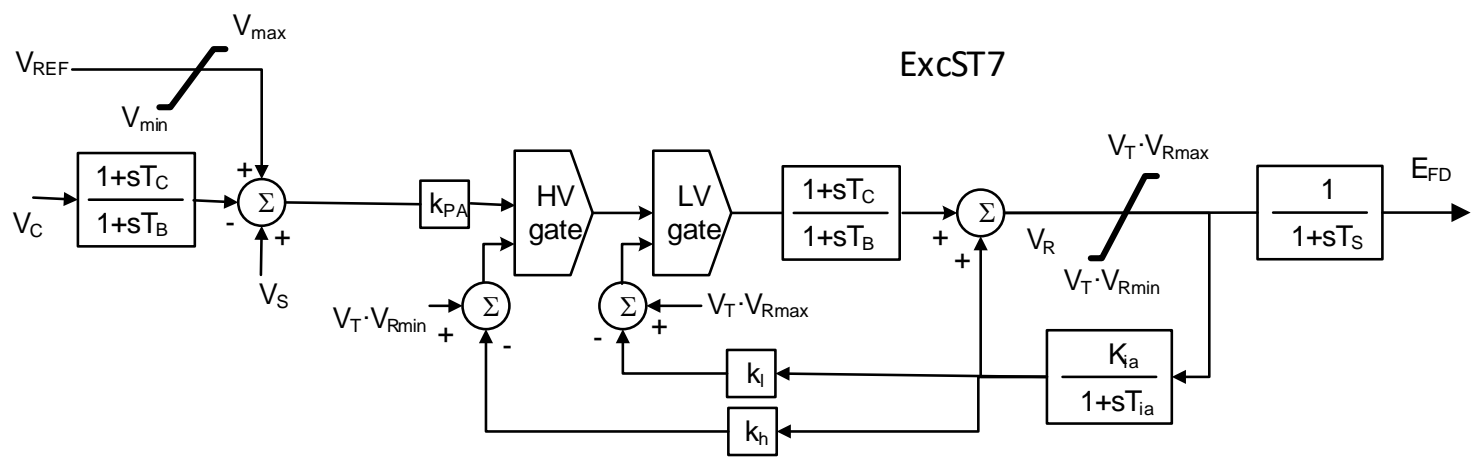

Fig. 11. Block diagram of ExcST7B model (simplified according to [4]).

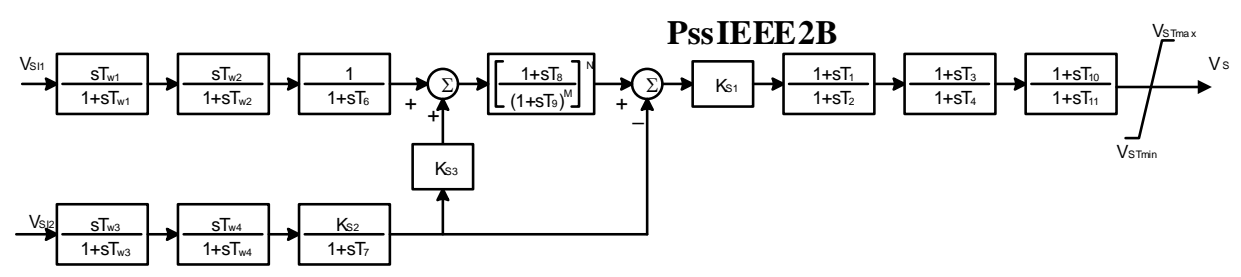

Power System Stabiliser

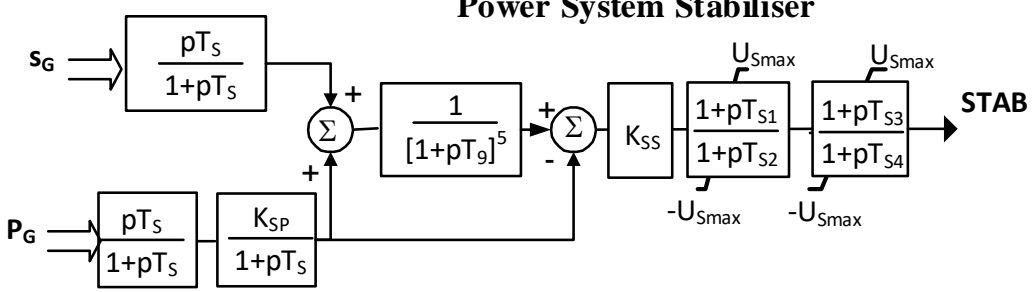

Fig. 12. Block diagram of PssIEEE2B model (upper part according to [5]) and system stabilizer from the MODES library.

\section{A. AC Generator Excitation System Model ExcAC6A}

The upper part of Fig. 9 gives the block diagram of ExcAC6A model according to CGMES. The diagram shown in the figure is simplified as the inputs of limiters are missing. The controller is proportional with the double lead-lag block. The AC output of the excitation system is rectified by an uncontrolled rectifier to DC input of the synchronous machine field winding. The terminal voltage unconventionally limits the output of the system controller. Thus ExcAC6A acquires dependent nature that is typical for the static excitation systems powered by auxiliary consumption. The model also comprises the limiter (highlighted in red) and the excitation voltage dependence on the unit speed $\omega$.

The model of the excitation system implemented in the network simulator MODES conceptually consists of the controller model and the exciter model itself representing the actuator. The controller model is actually common to all types of the excitation systems. The controller model comprises derivative feedback that is mainly used in DC excitation systems and $\mathrm{AC}$ excitation systems with the uncontrolled rectifiers. Moreover, the model includes the reactive current droop circuits (alternatively the active or reactive power). Separate models model the additional devices of the controller represented by yellow highlighted blocks. The controller output $R_{\mathrm{B}}$ is connected to the AC exciter model with an uncontrolled rectifier that is represented by AC1A model implemented in MODES. The output $R_{\mathrm{B}}$ may be limited by the terminal voltage $V_{\mathrm{G}}$ (for compatibility with the CGMES model ExcAC6A). MODES model does not allow simulating speed dependence.

\section{B. Static Potential-Source Excitation Model ExcST1A}

The block diagram depicted in the upper part of Fig. 10 is a simplified model of the excitation system ExcST1A according to CGMES. The proportional controller is modeled with the double lead-lag block. The terminal voltage of the synchronous unit limits the voltage of the controlled rectifier. Unconventionally, the model includes limiter (highlighted in red) and takes into account the voltage drop across reactance $X_{\mathrm{S}}$.

The corresponding model of the static potential-source excitation system ST_1 (implemented in MODES) considers upper limit of the voltage $U_{\mathrm{Bmax}}$ for the fully loaded rectifier. The limiter of the excitation current is shown as a block representing separate auxiliary circuit. ST_1 model is depicted in the lower part of Fig. 10.

\section{Static Potential-Source Excitation Model ExcST7B}

A simplification of the model ExcST7B according to CGMES is depicted in Fig. 11 (alternative inputs of the limiters $V_{\mathrm{UOL}}$ a $V_{\mathrm{OOL}}$ are missing). The transfer function of the controller may be written in the form $k_{\mathrm{PA}}\left(1+1 / \mathrm{s} T_{\mathrm{iA}}\right)$ under the condition $k_{\mathrm{ia}}=1$ and assuming that both limiters $\mathrm{E}_{\mathrm{FD}}\left(k_{\mathrm{I}}=k_{\mathrm{h}}=0\right)$ together with the lead-lag block $\left(T_{\mathrm{B}}=T_{\mathrm{C}}\right)$ are neglected. This results in the PI controller behavior.

The corresponding model in the simulator MODES is similar to the one shown in Fig. 10. The double lead-lag block is eliminated by assuming $T_{1}=T_{2}=T_{3}=T_{4}$ so the controller gains PI behavior with transfer function in the form $k_{\mathrm{P}}+1 / \mathrm{s} T_{\mathrm{I}}$ 


\section{Power System Stabilizer}

The device named as Power System Stabilizer (PSS) ensures fast damping of transient events. The parameter settings of PSS were the same for all mentioned excitation systems. The block diagram of the utilized PSS is shown in the upper part of Fig. 12, the model PssIEEE2B was selected. The model has a variability in the choice of input signals. However, the use of the speed deviation (generator slip) as the first input signal and active power of the generator as the second input signal $\left(P_{\mathrm{G}}\right)$ is the most common.

PSS is implemented as an auxiliary circuit of the excitation system controller in MODES. Its diagram is represented by the lower part of Fig. 12. It corresponds to the PssIEEE2B with several simplifications (simple washout blocks with time constant $T_{\mathrm{S}}$, zero parameter $T_{8}$ in the low-pass filter and only double lead-lag block $\left(T_{9}=T_{10}=0\right)$. Nevertheless, the fundamental properties of the system stabilizer are preserved as the higher frequencies of electromechanical oscillations are filtered off for the speed channel whereas active power channel is eliminated for lower frequencies of inter-area oscillations.

\section{Test CAse 3 - Simulation Results}

This part summarizes the results of dynamic simulations in MODES according to the test case 3. The following charts show the waveforms of the generator power $P_{\mathrm{G}}$, current $I_{\mathrm{G}}$ and terminal voltage $V_{\mathrm{G}}$ as well as the excitation voltage $E_{\mathrm{FD}}$.

\section{A. Model ExcAC6A}

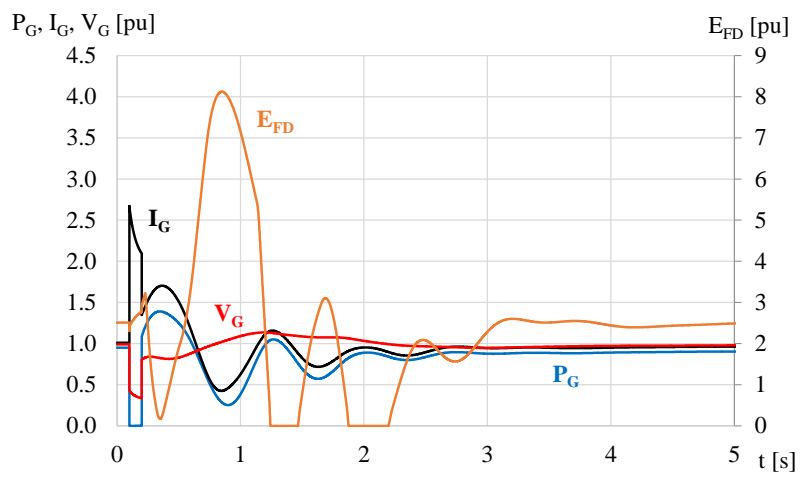

Fig. 13. Generator response to the short-circuit - ExcAC6A excitation.

Fig. 13 gives the results of time domain simulation according to the test case 3 for the unit with the ExcAC6A excitation system in operation. As can be seen, the quantities of the generator stabilize within 5 seconds.

\section{B. Model ExcST1A}

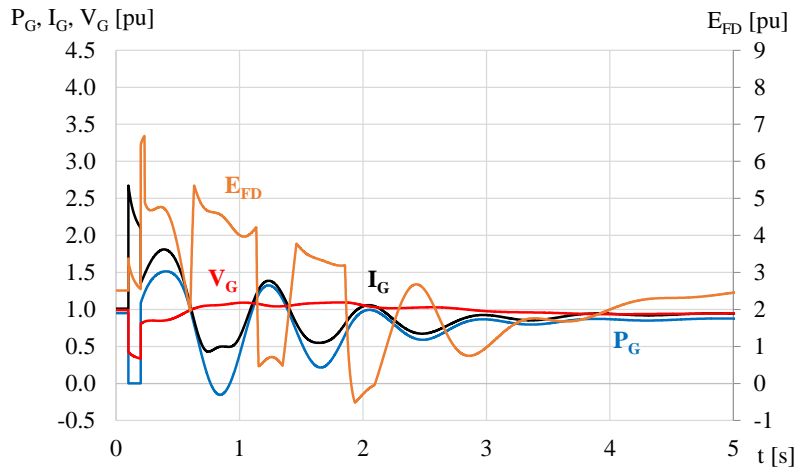

Fig. 14. Generator response to the short-circuit - ExcST1A excitation.
As it is evident in Fig. 14, the waveforms are stable. Unlike the previous model, the model ExcST1A allows field weakening, thus the excitation voltage $E_{\mathrm{FD}}$ goes into negative polarity.

\section{Model ExcST7B}

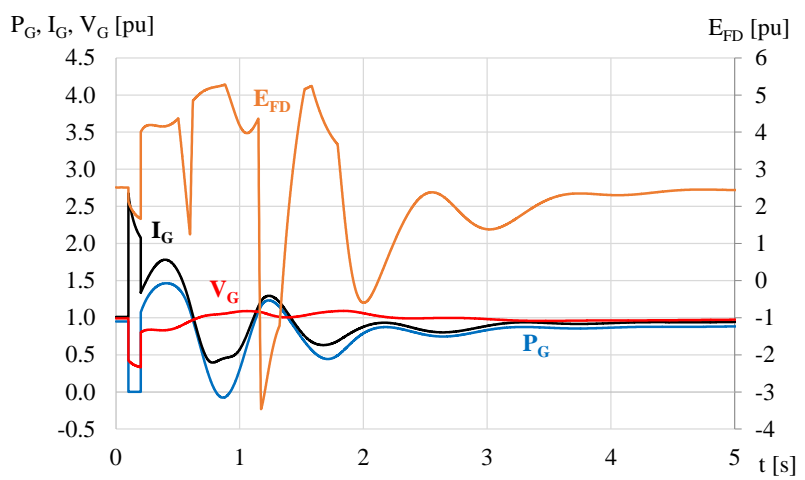

Fig. 15 Generator response to the short-circuit - ExcST7B excitation

Fig. 15 shows the response to three-phase short-circuit of the synchronous unit with the ExcST7B model.

The last figure compares time domain simulation of the generator slips $\left(S_{\mathrm{G}}\right)$ for each excitation system model. Except for the model ExcST1A, the transients are damped within three swings.

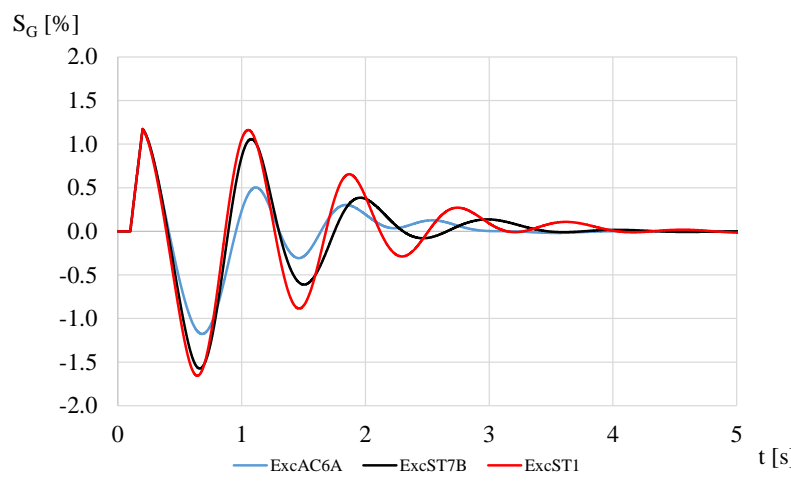

Fig. 16 The waveforms of generator slips for each excitation model

\section{CONCLUSION}

This paper deals with the generic turbine-governor and excitation system dynamic models. The paper compares the models from the CGMES standard, which is being used by ENTSO-E for the exchange of information among European transmission system operators, and the corresponding models implemented in the library of the network simulator MODES. The testing methodology defined by ENTSO-E SPD was applied to the models to reveal their dynamic response. The methodology includes time domain simulation while the system is being subjected to specific network disturbances like the island operation or short-circuit.

The European energy sector has undergone a significant transformation in the last decades. Unbundling, market opening as well as an implementation of new energy resources lead to increasing amount and distance of power exchanges together with power flow forecast uncertainty. Therefore, the power systems are operated closer to thermal and stability limits. The dynamic system behavior becomes more and more important for planning and operation of the transmission system. The dynamic 
behavior is investigated mostly with offline stability studies these days but the time frame of dynamic analyses has to evolve closer the real time with the mentioned changes in energy sector. The models presented in this paper are suitable for such studies. It is first necessary to verify that the models used in the stability studies are able to give credible results with help of elementary tests.

The transmission system of Continental Europe is interconnected system. Therefore, TSOs must perform coordinated dynamic stability assessment. However, each TSO may use different simulation tools. In order to provide comparable and credible results by the different simulation tools, it is necessary to ensure similar behavior of standard models. It is possible to use the mentioned elementary tests to detect the differences among the simulation tools and to tune model parameters if necessary. The contribution of the paper is that it contains the data for generic turbine-governor and excitation system models tested in other tools as well, so it is possible to compare the results with each other.

\section{REFERENCES}

[1] ENTSO-E, "Documentation on controller tests in test grid configurations", ENTSO-E SG SPD, 2013.

[2] K. Máslo, "Frequency stability in the context of the european network codes," in Proc. 9th International Scientific Symposium on Electrical Power Engineering ELEKTROENERGETIKA, 2017, pp. 436-442.

[3] K. Máslo and M. Kolcun, "Simulation engine for dispatcher training and engineering netw andork simulators," in Proc. IFAC CIGRE/CIRED Workshop on Control of Transmission and Distribution Smart Grids CTDSG, 2016, [On line]. Available: https://www.sciencedirect.com/science/article/pii/S2405896316323 $\underline{O O X}$

[4] ENTSO-E, Detail description of the CGMES profiles Version 2.4.14, [On line]. Available: https://www.entsoe.eu/majorprojects/common-information-model-cim/cim-for-grid-modelsexchange/cgmes-conformity/CGMES-v2.4.14/Pages/default.aspx

[5] IEEE Recommended Practice for Excitation System Models for Power System Stability Studies, in IEEE Std 421.5-2005 (Revision of IEEE Std 421.5-1992), pp.1-93, 21 April 2006 https://doi.org/10.1109/IEEESTD.2006.99499 